\title{
RoPod, a customizable toolkit for non-invasive root imaging, reveals cell type-specific dynamics of plant autophagy
}

\author{
Marjorie Guichard ${ }^{1,2,3, *}$, Sanjana Holla ${ }^{4}$, Daša Wernerová ${ }^{2}$, Guido Grossmann ${ }^{1,2,3}$ and Elena A. Minina ${ }^{1,4, *}$ \\ ${ }^{1}$ Centre for Organismal Studies, Heidelberg University, Im Neuenheimer Feld 230, 69120 Heidelberg, Germany \\ ${ }^{2}$ Institute of Cell and Interaction Biology, Heinrich-Heine-University Düsseldorf, 40225 Düsseldorf, Germany \\ ${ }^{3}$ CEPLAS Cluster of Excellence on Plant Sciences, Heinrich-Heine-University Düsseldorf, 40225 Düsseldorf, Germany \\ ${ }^{4}$ Department of Molecular Sciences, Uppsala BioCenter, Swedish University of Agricultural Sciences and Linnean Center for Plant \\ Biology, P.O. Box 7015, Uppsala, SE-750 07, Sweden \\ ${ }^{*}$ Correspondence: E.A. Minina: alena.minina@slu.se; M. Guichard: marjorie.guichard@hhu.de
}

\section{Summary}

Autophagy is the major catabolic process in eukaryotes and a key regulator of plant fitness. It enables rapid response to stress stimuli, essential for plastic adaptation of plants to changes in the environment. Fluorescent reporters and confocal microscopy are among the most frequently used methods for assessing plant autophagic activity. However, detection of dynamic changes in the pathway activity has been hampered by stresses imposed on living plant tissues during sample mounting and imaging.

Here we implemented RoPod, a toolkit optimized for minimally-invasive time-lapse imaging of Arabidopsis roots, to reveal a time-resolved response of plant autophagy to drug treatments typically used for pathway modulation and discovered previously overlooked cell type-specific changes in the pathway response. These results not only give an insight into the complex dynamics of plant autophagy, but also provide necessary information for choosing sampling time for the end-point assays currently employed in plant autophagy research.

RoPods are inexpensive and easy-to-use devices that are based on commercial or custom designed chambers, compatible with inverted microscopes. We describe a detailed protocol for the fabrication and use of RoPods and provide a complete pipeline including semi-automated image analysis for root hair growth assays, demonstrating the broader applicability of the RoPod toolkit.

\section{Keywords}

Arabidopsis, Minimal-stress conditions for fluorescence microscopy, Plant autophagy dynamics, Plant cell biology, Root hairs, Root microscopy, Time-lapse imaging.

\section{Introduction}

Autophagy, from Greek for "self-eating", is the major catabolic pathway conserved in all eukaryotes [1]. It allows cells to degrade superfluous or damaged content and upcycle products of degradation in the form of energy and building blocks. Autophagy is tightly linked to sensing availability of nutrients and serves as a gateway for multiple stress responses $[1,2]$. Plant autophagy is a key player regulating plant fecundity, longevity, biomass production, remobilization of nutrients, tolerance to pathogens and abiotic stress stimuli, and thus is a popular topic in fundamental and applied plant research [24].

Knowledge about the dynamic changes in autophagic activity is crucial for our understanding of its physiological roles. Autophagy has a strong catabolic capacity and thus its activity is tightly regulated to prevent runaway degradation of the cellular content. The dynamics of autophagy activation ${ }^{[5,6]}$ and oscillations of its activity ${ }^{[7]}$ have been studied on animal model organisms but plantspecific aspects have remained obscure until now. One of the factors impeding investigation of plant autophagy dynamics has been the lack of appropriate tools for it.

A large proportion of approaches used for studying plant autophagy relies on implementation of fluorescence microscopy ${ }^{[8,9]}$. Currently, such assays are invasive and likely to expose samples to unwanted stresses that might significantly impact autophagic activity, and thus skew the results. For example, mounting seedlings for fluorescence microscopy typically involves extensive mechanical stress, frequently followed by heat and drought stress induced by the use of a strong laser light and gradual evaporation of mounting medium during imaging. These factors limit duration of the assays and the number of biological replicates that can be imaged simultaneously. As a consequence, most of the currently available assays are end-point analyses and therefore do not provide sufficient information on the dynamics of the pathway. Furthermore, the 
bioRxiv preprint doi: https://doi.org/10.1101/2021.12.07.471480; this version posted December 9,2021 . The copyright holder for this preprint (which was not certified by peer review) is the author/funder, who has granted bioRxiv a license to display the preprint in perpetuity. It is made available under aCC-BY-NC-ND 4.0 International license.

type and severity of stress might also depend on the microscope hardware as reported in the study by Wang et al. [10]. Importantly, the abovedescribed drawbacks of live imaging have significant impact on all assays for plant stressrelated pathways.

For this study, we developed a protocol that significantly reduces stress during long-term imaging of Arabidopsis thaliana roots. Arabidopsis roots are an excellent system for plant cell biology studies, due to its predictable architecture, small size, transparency of cells and low autofluorescence. We provide a detailed protocol for growth and imaging of Arabidopsis roots in the RoPod, glass-bottom chambers optimized for timelapse advanced fluorescent microscopy. We showcase the advantage of the RoPod toolkit implementation during long-term observations of roots by assessing growth response of primary roots and root hairs to changes in the growth conditions.

We then illustrate the benefits of RoPod's use for studying stress-related pathways on example of plant autophagy. By implementing RoPod we were able to perform live-cell imaging of fluorescent reporters for plant autophagic activity, which provided us with the quantitative data at high temporal resolution. This approach finally revealed the complex dynamics of plant autophagic activity and demonstrated its cell type-specific kinetics.

\section{Results}

\section{Chambers and plant growth}

In order to enable minimally invasive and longterm time-lapse experiments, we developed an experimental setup that allows Arabidopsis seed germination and seedling growth in a device compatible with both plant growth and microscopy. We first established a protocol for the use of glassbottom chambers in long-term experiments. We tuned parameters to achieve optimal humidity, aeration, growth medium transparency, changes in the vector of and seed plating layout. To this end, we tested five different types of commercially available coverslip-bottom chambers from three different providers (see Materials and Methods) and $0.5 x$ Murashige \& Skoog standard growing medium (MS) solidified with either $0.2 \%, 0.4 \%$ or $0.8 \%$ Plant Agar (Duchefa) or Phytogel (Sigma). Under our conditions, the medium solidified with $0.8 \%$ Plant agar provided the best results: plants had normal phenotype and image quality was identical to the one obtained with usual seedling mounting protocol on microscopy glass. The optimized amount of medium per chamber well is described in Materials and Methods.

For plant growth, sterile seeds were placed on the top of the medium in the chambers (Fig. 1a). The chambers with germinating seeds were sealed individually, placed into a square Petri plate (Fig. 1c) which was also sealed and incubated horizontally for four days in the plant growth cabinet to allow roots to reach the bottom coverslip. After that the plate with the chambers was tilted by 45-90 degrees to guide the root growth along the cover slip. Depending on the seed batch age and quality, germination might be more or less synchronized, leading to the significant delay in growth of some roots and complicating choice of the time point for the change of the vector of gravity. We tested seed germination and root growth in chambers placed continuously under $30^{\circ}, 40^{\circ}$ or $45^{\circ}$ angle (exampled on the Fig. 1c). However, even in this case the root growth was not always following the desired pattern. Additionally, the above-described protocol was not compatible for imaging roots younger than five days, impeding studies at earlier developmental stages.

To circumvent these issues, we further optimized the growth protocol. Sterile scalpel or pipette tip was used to remove a strip of the medium from the chamber (Fig. 1d) and the seeds were placed on the coverslip bottom by the edge of the medium. The chambers were then incubated under $45^{\circ}$ angle to guide the root growth along the coverslip. This optimization significantly improved reliability in the root growth direction and enabled imaging of the roots as early as three days after seed plating.

All commercially available chambers we tested had significant drawbacks when used for plant growth. For example, it was not possible to perform longterm treatment using liquid in vertically placed commercially available chambers. This is particularly relevant for long-term experiments involving inducible promoters or drug applications that require extended treatment times as well as for experiments relying on image acquisition using a vertical imaging system such as the SPIRO (https://www.alyonaminina.org/spiro) or a vertical-mount microscope [11]. Furthermore, the chambers we tested were not designed for repeated use resulting in a complete or partial detachment of the coverslip from the chamber frame after several rounds of washing and sterilization. These limitations prompted us to design the 3D-printed RoPod chambers optimized for plant-specific use.

We designed a set of RoPod chambers (Fig. 1b, Supporting information File S1) with various number of wells and watertight features, optimal for a broad range of experiments and compatible with typical microscope stages. We tested various types of silicon glue, super glue and UV glue to optimize robustness of the chambers and ensure lack of phytotoxicity. To our experience superglue (Loctite, Precision Super Glue) provided the best results. However, we further improved on the 
a

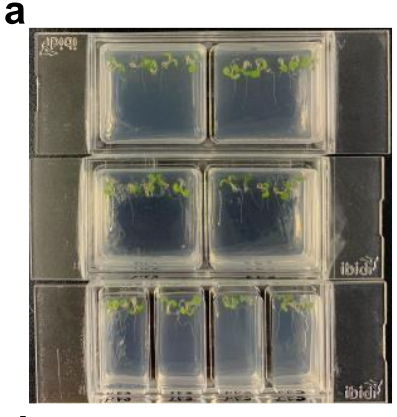

d

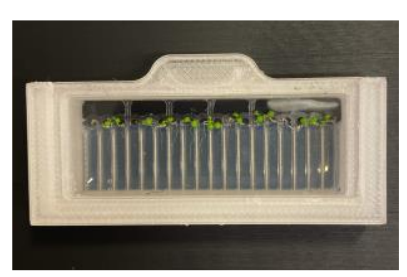

b

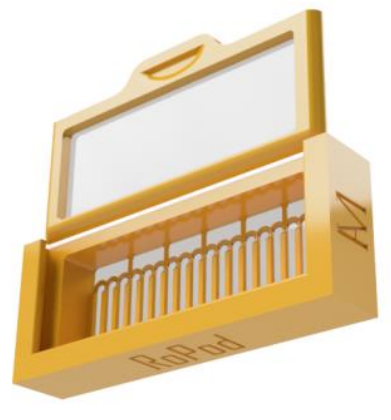

e

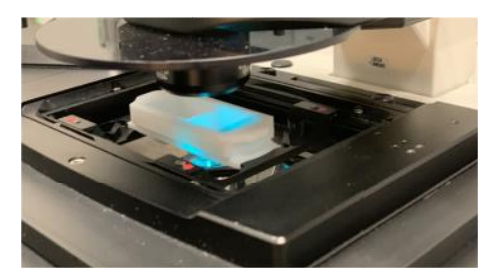

C

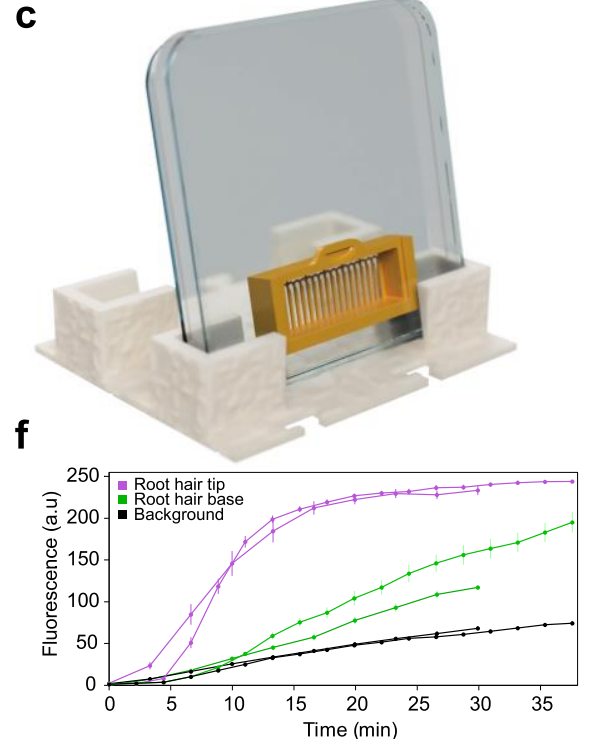

Figure 1. RoPod, a 3D printed system designed for microscopy on Arabidopsis roots. (a) A photo of Arabidopsis seedlings grown in commercially available chambers using RoPod protocol. Chambered coverslips (cat \# 80421 and 80286, Ibidi, Germany). (b) A rendering of the RoPod 5 optimized for growth and imaging of Arabidopsis. The chamber has plastic separators for each root and arcs to guide the young roots into individual lanes. Standard microscopy cover glasses are embedded in the plastic during printing, making the chambers robust and watertight and thus reusable and suitable for supplying liquid in the vertically positioned chambers. (c) A printable stand for a standard $12 \mathrm{~cm}$ square Petri plate has a 12 degrees tilt to direct root growth towards the glass bottom of the chambers. (d) Arabidopsis thaliana seedlings grown for 7 days in the chamber shown in b. (e) A chamber mounted on a confocal laser scanning microscope stage. (f) Diffusion of chemicals in RoPods exampled by accumulation of the green fluorescence in root hair cells of the plants grown in the chamber upon addition of fluorescein to the top of the medium. Data for two independent experiments is plotted for each type of sample quantification. For representative images and fluorescein diffusion rate in the chambers containing only medium see Supporting information Fig. S1.

chambers performance by designing RoPods with microscopy grade coverslips embedded in plastic during the printing process, rendering these chambers resistant to repeated cleaning and reusing (Fig. 1b-e). The watertight RoPod 5 chamber allowed us to perform long-term timelapse imaging of the range of several days, when scanning sessions were made overnight and during the day time chambers were placed vertically into the growth cabinets to maintain gravity vector and guide the root growth along the coverslip bottom. To enable easy tracking of the same biological replicates over multiple imaging sessions we designed a watertight RoPod 5 with separate lanes to guide growth of individual roots. The width of lanes was optimized to not impose mechanical stress on the growing roots and arcs steer growth of young roots into the lanes (Fig. 1b, Supporting information File S1).

To test whether roots grown in the RoPod are amenable to drug treatments, we assessed diffusion of chemical compounds in the medium inside RoPod chambers. We tracked diffusion rate of the fluorescent dye fluorescein in horizontally placed RoPods containing only growth medium (Supporting information Fig. S1) and in chambers with seedlings (Fig. 1f, Supporting information Fig. $\mathrm{S} 1)$. In the chambers containing medium only, we detected increase in fluorescence over time at the bottom cover slip, while in the chambers containing seedlings, we measured the fluorescence increase over time at root hair tips. Quantification revealed that the dye added on the surface of the growth medium diffuses to the bottom coverslip and reaches the plant tissue within approximately 15 25 min (Fig. 1f, Supporting information Fig. S1). Such treatment rates are well compatible with assessments of relatively slow dynamic responses (tens of minutes to hours), such as plant autophagy.

In summary, the RoPod toolkit is a simple to use solution for minimal-stress imaging of Arabidopsis roots. The optimized protocol for Arabidopsis growth in chambers is provided in the Supporting methods. The 3D printed RoPod chambers are customized for plant research and are comparable in price with the use of regular microscopy slide and coverslip but provide a great advantage of imaging under controlled conditions, thus enhancing experimental reproducibility.

\section{RoPod plant growth protocol overview}

The different functional designs of RoPod chambers, as well as an illustrated step-by-step protocol for their 3D printing are presented in Supporting information File S1 and Supporting methods. Updates of the designs and of the 
bioRxiv preprint doi: https://doi.org/10.1101/2021.12.07.471480; this version posted December 9,2021 . The copyright holder for this preprint (which was not certified by peer review) is the author/funder, who has granted bioRxiv a license to display the preprint in perpetuity. It is made available under aCC-BY-NC-ND 4.0 International license.

printing protocols depending on the model of 3D printer used are published on the website: https://www.alyonaminina.org/ropod. recommended protocol to grow seedlings in RoPods is presented in the Supporting methods and its modifications can be found in the Materials and Methods section.
Validation of RoPod applicability for low-stress long-term time-lapse imaging of Arabidopsis thaliana roots

After optimizing growth conditions and obtaining an overall wild-type-like phenotype of plants grown in chambers, we performed a more in-depth analysis of the physiological parameters for a

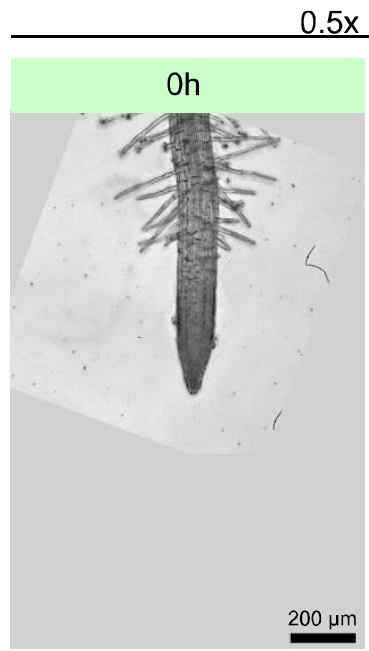

b

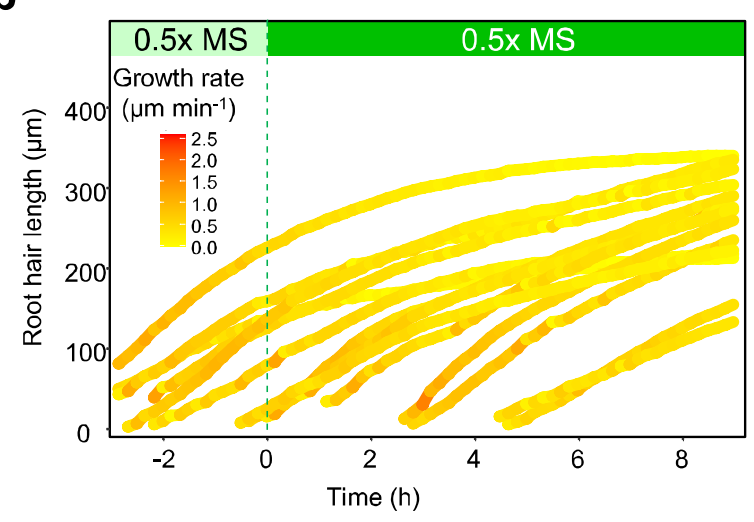

$0.5 \times \mathrm{MS}+1 \%$ sucrose
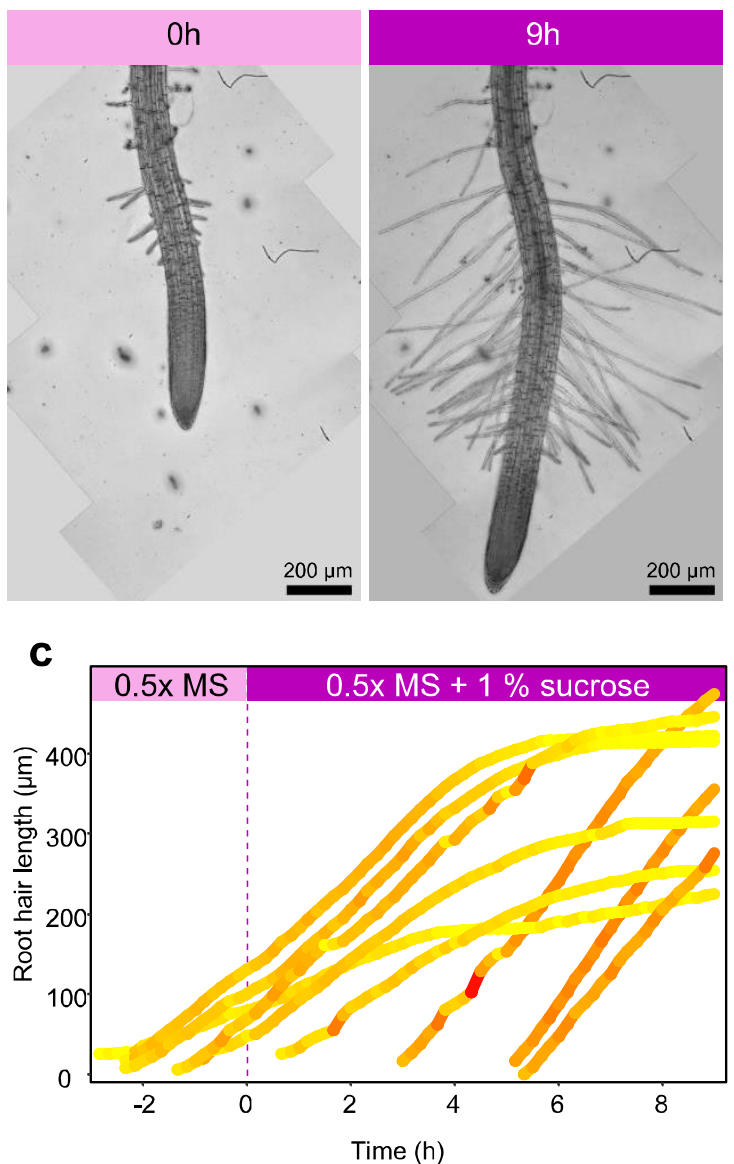

d

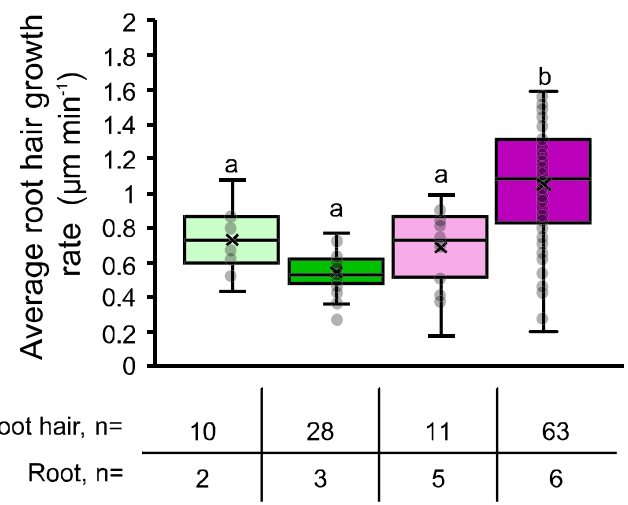

Figure 2. Seedlings grown and imaged in RoPods show expected root hair elongation rates under normal and sucrose-rich conditions. Arabidopsis Col-0 wild-type seedlings were grown for one week in a RoPod using the described protocol. The growth of root hairs was firstly recorded for $3 \mathrm{~h}$ under control conditions. After that, the chambers were flooded with liquid 0.5x MS (control treatment) or liquid $0.5 \times$ MS supplemented with $1 \%$ of sucrose (sucrose treatment). (a) Representative images showing root hairs at $0 \mathrm{~h}$ and $9 \mathrm{~h}$ of treatment with two different media. Each panel is a minimum intensity projection of $15 \mathrm{z}$ sections spaced $30 \mu \mathrm{m}$ apart. The root hair length was measured by tracking root hair tips of one representative root for control treatment (b) and for sucrose treatment (c). (d) The root hair growth rate was calculated during the linear growth phase, defined between $50 \mathrm{~min}$ after the emergence of the hair bulge and the time at which the hair does not grow more than $16 \mu \mathrm{m}$ for 40 consecutive minutes. The sample size used for each measurement is shown in the table below the chart. Kruskal-Wallis test with Dunn's multiple comparison test, $\mathrm{P}<0.05$. 
bioRxiv preprint doi: https://doi.org/10.1101/2021.12.07.471480; this version posted December 9, 2021. The copyright holder for this preprint (which was not certified by peer review) is the author/funder, who has granted bioRxiv a license to display the preprint in perpetuity. It is made available under aCC-BY-NC-ND 4.0 International license.

seedlings grown and imaged in RoPods.

Firstly, we assessed root growth rate in the RoPod chambers. For this, seeds of Arabidopsis Col-0 accession were plated in RoPod 5 chambers using the described protocol. The chambers were then mounted on a vertical imaging system SPIRO (https://www.alyonaminina.org/spiro), which imaged RoPods every 30 min for duration of two weeks. Growth of 20 roots in two RoPods was then measured manually using the obtained time-lapse images and the Fiji software. The average root growth rate in RoPods was $2.8+/-0.1 \mu \mathrm{m} \mathrm{min}{ }^{-1}$ (standard error), which is consistent with the growth rate observed under normal conditions [12]. A $6 \mathrm{~h}$ fragment of a time-lapse video is presented in video $\mathrm{S} 1$.

Next, we tested whether RoPods can provide lowstress conditions for long-term time-lapse imaging. For this, we used the rate of root hair growth as a sensor for stress conditions. Root hairs sustain a plethora of functions including nutrient uptake, plant anchoring in soil and symbiotic interactions (see Rongsawat et al., 2021 for review [13] ). Their growth rate is known to be affected by mechanical stress, fluctuations in humidity, temperature and to change depending on sucrose availability [14]. Under standard conditions, Arabidopsis root hairs elongate at the rate of ca. $1 \mu \mathrm{m} \mathrm{min}{ }^{-1}{ }^{[15]}$ and a typical root hair measures ca. $100-400 \mu \mathrm{m}$ depending on the growth conditions. Hence, the entire process of a root hair formation from initiation to termination spans several hours.

We chose an experimental setup that involved addition of medium supplemented with $1 \%$ sucrose during the course of the experiment to analyze hair growth under unperturbed conditions and upon treatments. By implementing RoPod, we recorded root hair growth within a time range of $9 \mathrm{~h}$, with a time resolution of $10 \mathrm{~min}$, for 16 roots and 112 root hairs in total under control and sucrose-rich conditions. To enable analysis of bulk imaging data, we developed a semi-automated image analysis pipeline involving designated Fiji macros (see Materials and Methods for details and https://github.com/AlyonaMinina/RoPod). This pipeline facilitated efficient analysis of the 6048 acquired images. Untreated root hairs grew up to $300 \mu \mathrm{m}$ in length at a rate of $0.5 \mu \mathrm{m} \mathrm{min}{ }^{-1}$. The treatment with sucrose increased the growth rate almost two fold (without sucrose: $0.54+/-0.02$ $\mu \mathrm{m} \mathrm{min} \mathrm{min}^{-1}$; with sucrose: $1.06+/-0.04 \mu \mathrm{m} \mathrm{min} \mathrm{m}^{-1}$ (mean +/-SE)) (Fig. 2, Video S2). Taken together, these results demonstrate suitability of RoPods to obtain stable growth conditions for long-term imaging at cellular resolution, as well as the possibility to apply treatments during the course of an experiment.

Since root hairs are often used as a model to study cell polarity in plants ${ }^{[16-19]}$, we provide a complete pipeline for root hair growth analysis under physiologically-relevant conditions. Although this protocol was originally developed for validating RoPods applicability it can be implemented for studies involving root hair analysis in the future.

Time-lapse imaging for tracking autophagic activity in Arabidopsis thaliana roots

Autophagy is a catabolic pathway that enables cells to degrade damaged or superfluous content and upcycle the products of degradation, keeping cell components in a functional state and also rendering cells less dependent on external sources of energy and building blocks [1,2,20]. Upon induction of autophagy a double membrane vesicle forms in the cytoplasm, engulfing cargo destined for degradation. Ripe plant autophagosomes are delivered to Iytic vacuoles, where they undergo hydrolysis. Autophagy is executed by autophagyrelated proteins, ATG, some of which are used as reporters for tracking the pathways' activity $[8,20]$. For example, under normal conditions, plant ATG8 protein localizes diffusely in the cytoplasm and nuclei. However, during induction of autophagy, ATG8 is incorporated into autophagosomes and trafficked to the lytic vacuole. Tracking changes in the localization of ATG8 from diffuse to puncta-like structures allows to assess formation of autophagosomes and also the rate of their delivery to vacuole, thus providing an estimation of autophagic activity $[8,9,21]$. Mostly due to the abovedescribed limitations of advanced fluorescence microscopy for living plant tissues, such experiments are performed using single time points. As a result, the pathway dynamics have remained obscure.

Here, we performed time-lapse tracking of ATG8 localization under low-stress imaging conditions. To this end, Arabidopsis thaliana seedlings expressing pHusion-tagged ATG8 (tandem tag consisting of GFP and RFP proteins ${ }^{[22]}$ ) in wild type or autophagy-deficient background were grown in the RoPod for 5-7 days using the protocol described in the Materials and Methods.

The basal level of autophagic activity typical for cells not subjected to stress was measured in cells of seedlings treated with $500 \mathrm{nM}$ concanamycin $A$ (ConA). ConA inhibits V-ATPase activity, causing neutralization of vacuolar $\mathrm{pH}$, deactivation of Iytic enzymes and consequently preservation of autophagic cargo that was delivered to the vacuole [8]. To artificially induce autophagic activity, we applied treatment with 500 nM AZD 8055 (AZD), an inhibitor of TORC1, a kinase complex that under normal conditions dampens autophagic activity $[8,21]$. The compound stocks were diluted in $0.5 \times \mathrm{MS}$ liquid medium and added to the RoPod chambers with seedlings immediately prior to scanning. Approximately $100 \mu \mathrm{m}$ deep z-stacks were acquired for each biological replicate every $15 \mathrm{~min}$. 
bioRxiv preprint doi: https://doi.org/10.1101/2021.12.07.471480; this version posted December 9, 2021. The copyright holder for this preprint (which was not certified by peer review) is the author/funder, who has granted bioRxiv a license to display the preprint in perpetuity. It is made available under aCC-BY-NC-ND 4.0 International license.

a

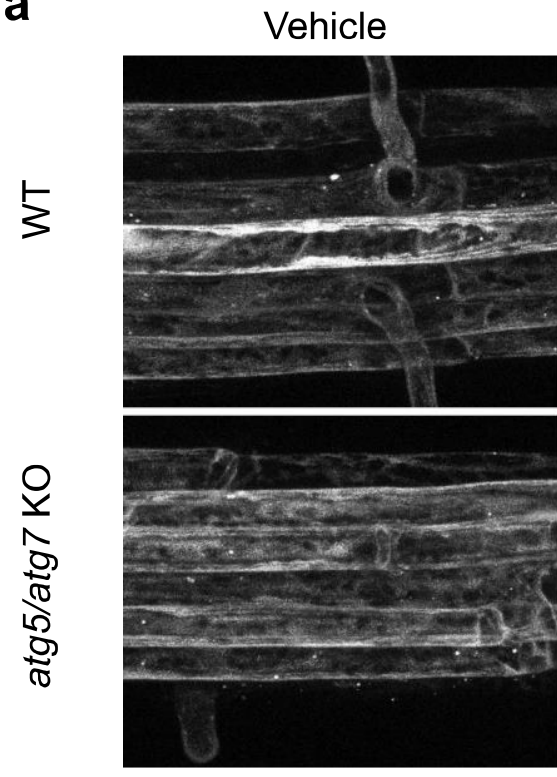

b

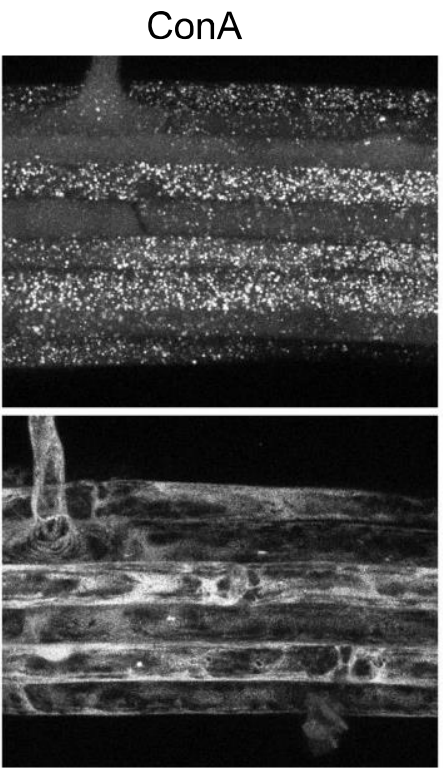

ConA

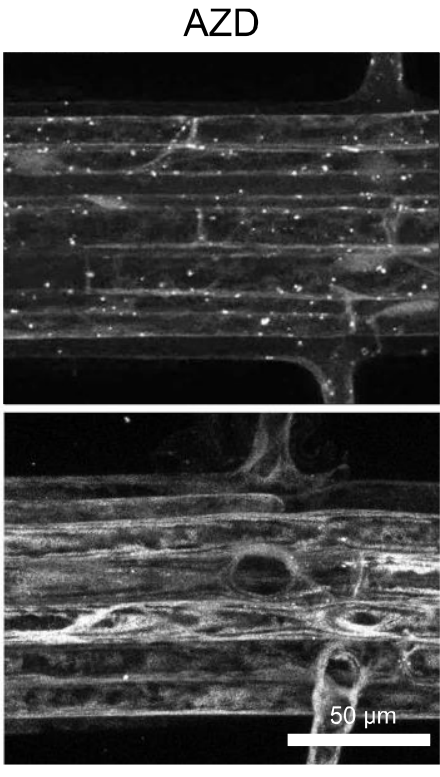

AZD
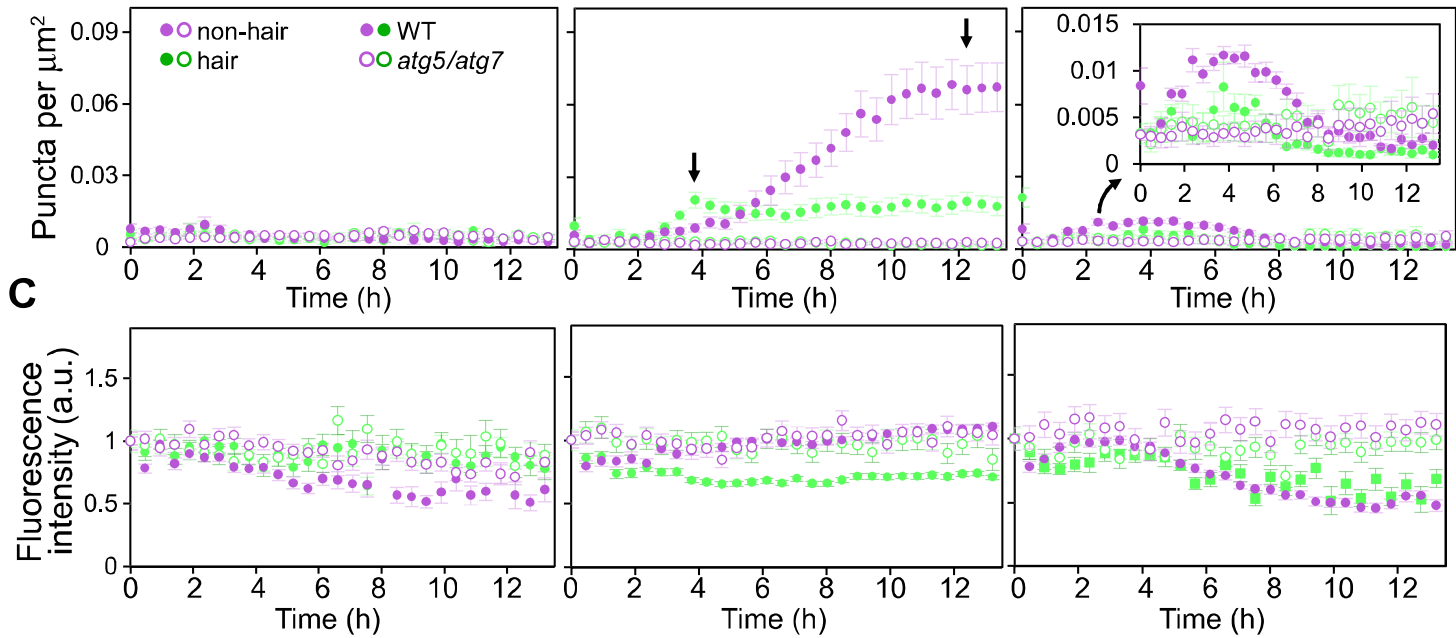

Figure 3. Time-resolved detection of plant autophagic activity provides a new insight on the pathway

functionality. Arabidopsis seedlings expressing autophagosomal marker pHusion-ATG8 in the wild-type (WT) or autophagy-deficient (atg5/atg7 KO) backgrounds were grown in the RoPods and treated with $0.5 \mu \mathrm{M}$ AZD8055 (AZD) to induce autophagy, or with $0.5 \mu \mathrm{m}$ concanamycin A (ConA) to inhibit degradation of autophagosomes delivered to the lytic vacuole. $0.1 \%$ DMSO (Vehicle) was used as a negative control for both treatments. The images were acquired at maximum 15 min intervals. Only GFP channel is shown. (a) Example time frames illustrating accumulation of pHusion-ATG8-labeled puncta in epidermal root cells upon treatment with DMSO, ConA and AZD. Complete time-lapse data is available in video S3. (b) Quantification of the number of puncta in the time-lapse data shown in video S3. The arrows in the ConA chart point out the peaks in cell-type specific autophagic activity. The inset on the AZD chart contains a zoomed view of the data indicated by the arrow. The dots depict mean \pm SE calculated with the number of puncta counted in each individual cell file. 2 to 15 cell files were analyzed for each sample, cell type and time point. Statistical analysis of puncta accumulation at $0 \mathrm{~h}, 4 \mathrm{~h}$ and $12 \mathrm{~h}$ in the hair and non-hair root cells is shown in Supporting information Fig. S2.(c) Quantification of GFP intensity for the data illustrated in video S3. The dots indicate mean \pm SE calculated with individual measurement of each punctum. The mean intensity has been corrected to subtract the background signal and normalized to the first time point of the record. Fluorescence intensity was measured for each vesicle, for a total population of 8 to 2339 vesicles per cell file, depending on the genotype, the treatment, the cell file type and the time of the treatment. 
bioRxiv preprint doi: https://doi.org/10.1101/2021.12.07.471480; this version posted December 9,2021 . The copyright holder for this preprint (which was not certified by peer review) is the author/funder, who has granted bioRxiv a license to display the preprint in perpetuity. It is made available under aCC-BY-NC-ND 4.0 International license.

Maximum intensity projections of the optical slices for each position at each time point were used for further image analysis. Representative images of pHusion-ATG8 localization at approx. $8 \mathrm{~h}$ (for ConA) and $3 \mathrm{~h}$ (for AZD) of the treatment are shown (Fig. 3a). Complete time series is available in video S3.

ConA inhibits degradation of autophagosomes and allows to quantify their number during the basal housekeeping autophagy occurring under normal conditions. Time-lapse imaging using RoPods confirmed previously observed differences in autophagic activity in hair and non-hair epidermal root cells of wild-type plants ${ }^{[21]}$. Namely, ConA treatment shorter than $5 \mathrm{~h}$ resulted in the higher amount of the pHusion-ATG8-positive puncta accumulating in the vacuoles of the hair root cells (Fig. 3b, Supporting information Fig. S2). Unexpectedly, at later time points, the number of puncta in hair cells plateaued, while in non-hair cells it increased more than threefold (Fig. 3b, arrows on the ConA chart, Video S3, Supporting information Fig. S2). This strong accumulation of pHusion-ATG8-positive puncta at later time points could be a consequence of stress caused by prolonged exposure to ConA. However, the cell type-specific pattern strongly indicates differences in autophagic pathway regulation in hair and nonhair root cells. We confirmed that observed changes in localization of pHusion-ATG8 were autophagy-specific by demonstrating no induced formation of pHusion-ATG8 puncta and no discernable differences between hair and non-hair cells in autophagy-deficient atg5-1/7-2 mutant seedlings (Fig. 3). Viability of cells was confirmed by the normal rate of elongation and root hair formation during imaging (Video S3).

Consistently with the results obtained on ConA treated roots, treatment with AZD also revealed a stronger upregulation of autophagic activity in the non-hair cells, although at earlier stages of treatment (Fig. 3b). This indicates that non-hair cells might possess higher capacity not only for basal autophagy but also for stress-induced autophagic activity.

Importantly, implementation of the time-lapse imaging using RoPods clearly illustrated how quantification of puncta induced by AZD treatment might provide erroneous results if made at a suboptimal time point. Namely, quantification of puncta shown in Fig. 3b demonstrates that their number is not different in the wild-type and autophagy-deficient background after ca. $6 \mathrm{~h}$ of AZD treatment. Such lack of difference can be explained by degradation of the autophagy reporter triggered in the wild-type cells, which would eventually lead to decrease in detectability of the signal. Indeed, after $4 \mathrm{~h}$ of treatment, the mean intensity of the fluorescent marker becomes significantly lower in the cells of wild-type plants compared to the ATG knockout cells (Fig. 3c), rendering comparison of the data from these two types of samples not reliable.

In summary, we demonstrate that plant autophagic activity fluctuates in a cell typedependent manner. We show that basal autophagic activity is present at different levels in hair and non-hair root cells. Similarly, induced autophagic activity also varies depending on the cell type. These observations lay ground for more detailed investigations of cell type-specific roles of autophagy in plant physiology.

We illustrate implementation of RoPod as a robust tool for studying plant autophagy dynamics and provide time-resolved data for the commonly used modulators of plant autophagy. This information will guide future analyses, highlighting the need for time-lapse studies in order to accurately interpret the role of autophagy in plant physiology and will serve as an important insight during selection of time points for the conventionally used end-point assays.

\section{Discussion}

In this study we demonstrate the benefits of the RoPod toolkit optimized for studying stressresponse related pathways in plants and provide a detailed protocol for its implementation.

An important advantage of RoPod over the previously published solutions ${ }^{[23]}$ is that in our toolkit plants are grown and imaged within the same chamber containing growth medium. Thus, seedlings are subjected to minimal changes in conditions when moved from growth cabinet to microscope stage and back. The most prominent advantage of the RoPod-based imaging is its applicability for long-term time-lapse data acquisition. It eliminates the typical issue with mounting medium evaporation during prolonged imaging and aids dissipation of the heat from laser light used for confocal microscopy. We validate the efficacy of RoPods in providing low-stress conditions during long-term imaging by tracking root growth rate and elongation rate of root hairs under control and sucrose-rich conditions.

We designed a watertight RoPod 5 chamber with arc-shaped guides and separators for roots of individual seedlings. Such design allows performing long-term experiments that include drug treatments dissolved in liquid medium and is compatible with moving the chamber between the microscope stage and a growth cabinet, while still being able to easily track the same biological replicates. To aid setup of such experiments, we provide experimental data on chemical diffusion rates within RoPod chambers.

The 3D-printed RoPods are easy to produce, very 
bioRxiv preprint doi: https://doi.org/10.1101/2021.12.07.471480; this version posted December 9 , 2021. The copyright holder for this preprint (which was not certified by peer review) is the author/funder, who has granted bioRxiv a license to display the preprint in perpetuity. It is made available under ACC-BY-NC-ND 4.0 International license.

robust and comparable in costs to standard microscopy glass for mounting samples, while providing a significant improvement in physiological relevance of the data and its quality. RoPods assembly does not require training or complex infrastructure, for instance, printing can be done at public Makerspace facilities available around the world. We designed RoPods to be compatible with commonly used microscopy equipment and applicable for a broad range of experiments, making them a great addition to more sophisticated solutions such as microfluidic chambers $[12,24]$.

By implementing the RoPod toolkit, we provide, for the first time, data on the dynamics of plant autophagy detected in root epidermal cells and show cell type-specific differences in their kinetics. Time-lapse detection of ATG8 in plant cells upon AZD-treatment illustrated the drastic impact of the reporter degradation via autophagy on the readout of the assay. We demonstrate that RoPod toolkit allows to estimate the optimal time range for quantification of the reporter under various conditions. The time-resolved data on autophagic activity obtained in this study can be further used as a guideline for accurate interpretation of the commonly used end-point assays for plant autophagic activity and can serve as a basis for detailed investigation of cell type-specific functions of plant autophagy.

\section{Materials and methods}

\section{Genetic constructs}

pMDC pHusion 1 Gateway vector was generated by amplifying pHusion tag from the p16:SYP61pHusion construct [22] using primers TTGGTACCATGGCCTCCTCCGAGGACG and TTGGCGCGCCCCCCTTGTACAGCTCGTCCATG and introducing amplicon into pMDC32 vector ${ }^{[25]}$ via $\mathrm{KpnI} / \mathrm{AscI}$ restriction digestion sites.

AtATG8a CDS Gateway entry clone [21] was then recombined with the pMDC pHusion 1 Gateway vector to obtain the construct driving expression of pHusion-AtATG8a under control of double $35 \mathrm{~S}$ promoter.

\section{Plant material}

Col-0 wild-type plants were transformed using GV3101 strain of Agrobacterium tumefaciens carrying pMDC pHusion1 AtATG8a construct as described in ${ }^{[21]}$. To obtain the autophagy-deficient control line, the established reporter line was crossed with atg5-1/atg7-2 double knockout plants.

\section{Microscopy chambers}

In this study we tested five types of microscopy coverslip bottom chambers from three different providers: Ibidi chambered coverslips (cat \# 80421 and 80286 \#1.5 polymer coverslip, hydrophobic, sterilized, Ibidi, Germany), 1-well II

\begin{abstract}
Chamber Slide ${ }^{\mathrm{TM}}$ System (Nunc ${ }^{\mathrm{TM}}$ Lab-Tek $^{\mathrm{TM}}$, cat \# C6307, Sigma-Aldrich), $x$-well cell culture chamber, 1-well, on coverglass (cat \# 94.6190.102, Sarstedt).
\end{abstract}

We then designed customized 3D printable chambers optimized specifically for growth and imaging of Arabidopsis roots. Printing of the chambers was tested using Prusa MK2.5S, MK3S, MK3S+ and Ultimaker S5 3D printers. For printing and assembly instructions see the Supporting information File S1 and Supporting methods.

We used 0.5x MS medium with vitamins (M0222, Duchefa), supplemented with 10 mM MES (M1503, Duchefa), $1 \%$ sucrose; for solid medium $0.8 \%$ Plant agar (P1001, Duchefa), pH 5.8 adjusted with $\mathrm{KOH}$. The medium was autoclaved at $120^{\circ} \mathrm{C}$ for 20 min. After autoclaving the medium was cooled to ca. $60{ }^{\circ} \mathrm{C}$ and pipetted into the chambers under sterile conditions: $0.75 \mathrm{ml}$ of medium per well of a 4-well chamber, $1 \mathrm{ml}$ of medium per well of the 3 -well chamber, $1.5 \mathrm{ml}$ per well of a 2 -well chamber and $3 \mathrm{ml}$ per 1 -well chamber.

The chambers were then individually sealed using plastic wrap (watertight RoPod chambers did not require this step), put into $12 \mathrm{~cm}$ square Petri plates that were sealed as well and placed into a growth cabinet.

To reuse the chambers, the growth medium was gently removed using a Q-tip, the chambers were washed with cold water and dish soap, rinsed with MilliQ water, air dried and sterilized for $30 \mathrm{~min}$ under UV light.

Fluorescein diffusion assay

For the diffusion assay in RoPod containing plants, Arabidopsis Col-0 seeds were surface sterilised in opened $2 \mathrm{ml}$ tubes for $2 \mathrm{~h}$ in an air-tight box containing chlorine gas, produced by the mix of 50 $\mathrm{ml}$ of sodium hypochlorite $10 \%$ (Roth, ref. 9062.3) and $2 \mathrm{ml}$ of $37 \%$ hydrochloric acid (Merck, ref. 1.00317.1000). Seeds were then aerated for 10 min in a sterile bench to remove the left-over chlorine gas. Seeds were then transferred, with a sterile toothpick, in 2-wells Ibidi chambers filled with $1 \mathrm{~mL}$ of growth medium in each well (0.5x MS (Serva, ref. 47515); MES $5 \mathrm{mM}$ (Sigma, ref. $\mathrm{M} 8250) ; 1 \%$ sucrose; $\mathrm{pH} 5.7$ adjusted with $\mathrm{KOH}$; $0.8 \%$ Plant agar (Duchefa, ref. M1002)). The RoPods were cultivated for eight days horizontally at $21^{\circ} \mathrm{C}, 16 \mathrm{~h}$ illumination, $100 \mu \mathrm{mol} \mathrm{m} \mathrm{m}^{-2} \mathrm{~s}^{-1}$. Then, the RoPods were incubated at $45^{\circ}$ for one day before image acquisition. At the start of the recording, each well containing plants was filled with $1 \mathrm{ml}$ of fluorescein at $4 \mu \mathrm{g} \mathrm{ml}^{-1}$ diluted in a liquid $0.5 x$ MS (final concentration after diffusion: $2 \mu \mathrm{g} \mathrm{ml}^{-1}$ ). Imaging was performed using Leica DMI8 confocal microscope and HC PL APO CS2 20x objective (ex, $488 \mathrm{~nm}$, em, $498 \mathrm{~nm}$ - $550 \mathrm{~nm}$, pinhole size of 1 Airy Unit) (Leica Microsystems, 

perpetuity. It is made available under aCC-BY-NC-ND 4.0 International license.

Germany), pixel dimensions $0.48 \mu \mathrm{m} \mathrm{px}^{-1}$, image size 512 x 512 px, 8-bit. The root hairs that were growing at the beginning of the recording were selected for analysis. To obtain a complete view of the root hairs, a z-stack was performed to cover 92 $\mu \mathrm{m}$ height with each section separated with $10 \mu \mathrm{m}$. The images were then processed on Fiji [26] to obtain maximum intensity projection. A circular region of interest ( $R O I$ ) with a $12 \mu \mathrm{m}$ diameter was used to measure the mean grey value on the selected root hair tips, on the base of the corresponding root hair tip and in the upper left corner of the image for the mean grey value of background. The experiment was performed in two individual boxes containing 2 to 3 roots each, 2 to 8 root hairs analyzed per root.

For the fluorescein diffusion assay in the solid medium and without plants, 2-wells Ibidi chambers filled with $1 \mathrm{ml}$ of growth medium in each well were used (0.5x MS (Serva, ref. 47515); MES $5 \mathrm{mM}$ (Sigma, ref. M8250); 1 \% sucrose; pH 5.7 adjusted with $\mathrm{KOH} ; 0.8 \%$ Plant agar (Duchefa, ref. M1002)). $1 \mathrm{ml}$ of liquid growth medium supplemented with $140 \mu \mathrm{g} \mathrm{ml} \mathrm{m}^{-1}$ of fluorescein solution was added at the start of the recording (final concentration after diffusion: $70 \mu \mathrm{g} \mathrm{ml} \mathrm{m}^{-1}$ ). The same microscope settings as for the previous diffusion assay were used (pixel dimensions 1.14 $\mu \mathrm{m} \mathrm{px}{ }^{-1}$, image size $512 \times 512 \mathrm{px}, 8$-bit). The fluorescence was recorded in 4 RoPods, with 2 to 3 fields of view chosen at random positions in each box. Fiji software was used for image analysis. An orthogonal projection was carried out in the middle of each acquisition area with the Orthogonal view tool. To define the bottom of the RoPod, the orthogonal view of the image acquired $2 \mathrm{~h}$ after the start of treatment was used. A rectangular ROI with the width of the orthogonal projection image and $10 \mu \mathrm{m}$ in height was drawn at the bottom of the RoPod chamber. This ROI was reused to measure the mean grey value on the orthogonal view for each time point recorded at the same location.

Plant growth and image analysis for autophagic activity assay

Growth medium (0.5x MS (Duchefa, ref. M0222); MES 10 mM (Duchefa, ref. M1503); 1 \% sucrose; pH 5.8; $0.8 \%$ Plant agar (Duchefa, ref. P1001)) was autoclaved for $20 \mathrm{~min}$ at $120{ }^{\circ} \mathrm{C}$. After autoclaving the medium was cooled to ca. $60{ }^{\circ} \mathrm{C}$ and pipetted into 4-wells Ibidi chambers under sterile conditions. $1 \mathrm{ml}$ of medium was used per well.

Arabidopsis thaliana seeds were surface sterilized in $70 \% \mathrm{EtOH}, 0.05 \%$ TritonX-100 for $20 \mathrm{~min}$, washed three times with $96 \% \mathrm{EtOH}$, air dried in a sterile bench and transferred into the chambers using a sterile toothpick. The chambers were then placed under long day growth conditions (16 h, 150 $\mu \mathrm{mol} \mathrm{m}^{-2} \mathrm{~s}^{-1}$ light at $22^{\circ} \mathrm{C}, 8 \mathrm{~h}$ dark at $20^{\circ} \mathrm{C}$ ). Typically, for the first four days the chambers were kept horizontally allowing the roots to reach the bottom cover slip. After this, the chambers were placed vertically to guide the root growth along the cover slip.

Drugs were diluted in $0.5 x \mathrm{MS}$ liquid medium (the same composition as the growth medium, but lacking Plant agar) to a final concentration of $1 \mu \mathrm{M}$ AZD8055 (Sigma, ref. ADV465749178, CAS

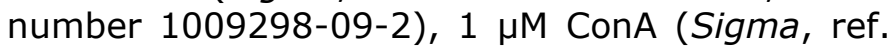
C9705, CAS number 80890-47-7) or 0.1 \% DMSO for the vehicle control. The solutions were pipetted into the wells of the chambers mounted on the confocal stage in $1: 1$ ratio ( 1 volume of the medium in the well: 1 volume of the drug solution. Drug final concentration: $500 \mathrm{nM}$ ) right before the start of imaging.

Imaging was performed using confocal laser scanning microscope (CLSM) Leica SP5 or SP8 (Leica Microsystems, Germany), 63x water immersion objective, $N A=1.2$, excitation light 488 $\mathrm{nm}$, emission range of $498 \mathrm{~nm}-541 \mathrm{~nm}$ and system-optimized pinhole size. Four to six biological replicates were imaged for WT and atg $\mathrm{KO}$ backgrounds in each experiment. The experiment was performed three times. Images were acquired at the beginning of the root differentiation zone, collecting a ca. $100 \mu \mathrm{m}$ deep z-stack starting at the surface of a root (pixel dimensions $0.29 \mu \mathrm{m} \mathrm{px}^{-1}$, image size $848 \times 848 \mathrm{px}$, 8 -bit). Each replicate was imaged every 10 to 28 min for a period of 12 to $18 \mathrm{~h}$. The growth settings were tested in two independent laboratories using Leica SP5, SP8 and Zeiss CLSM 800 microscopes. The optimized protocol for the plant growth is described in the Supporting methods.

Raw confocal image stacks were imported into Fiji [26]; a maximum-intensity projection of the GFP channel was generated, the data set was rotated to orient the root horizontally and cropped. The data set was registered using the MultiStackReg plugin with "rigid body" settings, starting at the final slide to correct for root growth and specimen movement during imaging. The registered data sets were cropped again to the maximum area available for continuous analysis throughout the time course. ROIs were defined along hair and nonhair cells, excluding cell borders, and data sets were exported as TIFF stacks for further analysis. Autophagosomes were then identified using pixel classification approaches with the software Ilastik [27]. The classifier was trained on five images (Col0 roots treated with: DMSO, ConA, AZD; and two atg5-1/atg7-2 roots treated with AZD). Pixel features analyzed by the software were defined according to their intensity (Gaussian smoothing with a radius of 0.3 and 0.7 pixel) and their edges (Laplacian Gaussian with of 0.7 and 1.0, and 

perpetuity. It is made available under aCC-BY-NC-ND 4.0 International license.

Gaussian Gradient Magnitude) with radius of 0.7 ). Three classes were defined for the training: vesicles, root and background. The classifier was trained by manually brushing areas of interest for each defined class. The simple segmentation produced was exported as 8-bit images in order to be processed further. In Fiji, regions of interest were defined to identify the hair and non-hair cell files to analyze. For each cell file ROI, its size, vesicles number, vesicles fluorescence intensity and vesicles size were quantified using an algorithm run in Fiji (Supporting information File S2). The number of vesicles was counted for each cell file type using data obtained from two independent experiments. 1 to 5 roots and 2 to 15 cell files per type were analyzed in total. The fluorescence intensity was measured for each vesicle counted, for a total population per cell file of 8 to 2339 vesicles, depending on the genotype, the treatment, the cell file type and the time of the record considered. The mean intensity has been corrected with the background signal and normalized to the first time point of the record. As the image acquisition rate varied between experiments due to different number of biological replicates, each presented time point actually corresponds to an interval of up to $15 \mathrm{~min}$.

Plant growth and image analysis of sucrose treatment assay

Arabidopsis thaliana Col-0 wild-type seeds were surface sterilized with the chlorine gas method (see the Fluorescein section of Material and methods). The seedlings were grown in Sarsted single well boxes (ref. 94.6190.102) filled with $1.75 \mathrm{~mL}$ of growth medium (0.5x MS (Serva, ref. 47515); MES $5 \mathrm{mM}$ (Sigma, ref. M8250); pH 5.7 adjusted with $\mathrm{KOH} ; 0.8 \%$ Plant agar (Duchefa, ref. M1002)). The RoPods were incubated for four days horizontally at $21^{\circ} \mathrm{C}$ with $16 \mathrm{~h}$ of light, $100 \mu \mathrm{mol} \mathrm{m} \mathrm{m}^{-2} \mathrm{~s}^{-1}$. They were then tilted at $45 \circ$ for three days. The recording was made with a Nikon Eclipse Ti microscope, Nikon S Fluor 10x DIC N1 objective, Hamamatsu camera (C11440), bright field. A basal line was recorded for $3 \mathrm{~h}$ before treatment in the control and treated RoPods. Then, $3 \mathrm{ml}$ of $0.5 x \mathrm{MS}$ liquid supplemented with $1 \%$ sucrose were added (final concentration: $0.63 \%$ ) and a control specimen was treated with $0.5 x$ MS without sucrose. Root hair growth was recorded for additional $9 \mathrm{~h}$. For each root, two to three consecutive $X Y$ fields of view were recorded to obtain images of the root hairs at different stages of development throughout the recording time (pixel dimensions $0.59 \mu \mathrm{m} \mathrm{px}^{-1}$, image size $1920 \mathrm{x}$ 1200 px, 16-bit). In addition, a z-stack encompassing $240 \mu \mathrm{m}$ with optical sections of 30 $\mu \mathrm{m}$ was carried out to obtain a clear view of the entire root hairs. Finally, an image was recorded every $10 \mathrm{~min}$. The images were analyzed with Fiji software. As the root hair shaft appears dark, a Z projection based on the minimum intensity was performed for each field of view. The corresponding field of view of each root was then stitched with the "3D Stitching" plugin. The hairs to be analyzed were then selected by drawing straight lines starting from their base and extending over the entire length of each of them (Supporting information Fig. S3). A Fiji macro to guide this step is available (Supporting information File S3, Video S4, https://github.com/AlyonaMinina/RoPod). Then, an algorithm run in Fiji was used to facilitate the tracking of the tip of the hair (Supporting information File S4, Fig. S3, Video S4, https://github.com/AlyonaMinina/RoPod). This algorithm consists in using the straight lines defined previously and the Straighten tool to rotate the root hair in a horizontal position, the tip facing the right side of the image (Supporting information Fig. S3). Then, a midline is drawn by the user throughout the hair (Supporting information Fig. S3). This midline is used to create a mask around it in order to prevent other hairs from being processed by the algorithm (Supporting information Fig. S3). A threshold is applied with the Auto-local Threshold tool (Phansalkar method radius 30 ) to obtain a white image of the root hair on a black background (Supporting information Fig. S3). The white pixel positioned in the rightmost part of the image is considered to be the tip of the hair (Supporting information Fig. S3). It is then important that the background is kept clear on the rightmost part of the root hair tip. For that purpose, a rough manual cleaning of the background is proposed in the algorithm (Supporting information Fig. S3). This method is not suitable for monitoring hair growth for the first few minutes after the root hair bulge emergence. This is due to the thresholding method which does not differentiate between the root hair and the root corpus. For this reason, a manual adjustment step is proposed to correct any errors (Supporting information Fig. S3). At the beginning of root hair growth, positions of bulges and young root hairs drift as the root finishes its elongation. Therefore, the absolute coordinates of the root hair tip between two time points do not only reflect the displacement of the root hair. To correct this drift, the coordinates of the root hair tip were projected orthogonally on the medium line previously drawn by the user (Supporting information Fig. S3). The coordinates of the projected root hair tips were then used to calculate root hair displacement over time. All the data were exported into a table saved in .xls format and the root hair tip tracks were saved as ROI files. For each tested condition, 2 to 6 roots were analyzed, with 10 to 63 hairs analyzed in total. To test these macros, some example images are provided in the Supporting information File S5. 
bioRxiv preprint doi: https://doi.org/10.1101/2021.12.07.471480; this version posted December 9,2021 . The copyright holder for this preprint (which was not certified by peer review) is the author/funder, who has granted bioRxiv a license to display the preprint in perpetuity. It is made available under aCC-BY-NC-ND 4.0 International license.

\section{Author contributions}

A.M. conceived the project and the RoPod design; M.G. and G.G. contributed experimental ideas. A.M. and M.G. performed the experiments. A.M., M.G. and G.G. analyzed the data. A.M., M.G. and G.G. wrote the manuscript. D.W. and S.H. contributed to optimizing chamber design, 3D printing and imaging conditions.

\section{Acknowledgments}

The authors are thankful to Prof. Dr. Karin Schumacher (Centre for Organismal Studies, Heidelberg University) for hosting A.M. during the development of this project and her helpful advice. The authors gratefully acknowledge financial support by a Marie Curie fellowship (MAPoPHAGY, 799433) to A.M., by a Heisenberg Professorship and a research grant by the Deutsche Forschungsgemeinschaft (DFG, GR 4559/4-1, GR 4559/5-1) and funds by Germany's Excellence Strategy (EXC-2048/1, project ID 390686111) to G.G.

\section{Competing interests}

The authors declare no competing interests.

\section{Supporting information}

Video S1. Arabidopsis seedlings grown in RoPod have normal phenotype and root elongation rate.

This time-lapse video shows seeds germination and seedlings growth in the RoPod 5 . Side by side growth of atg5-1/7-2 autophagy-deficient plants (nine seedlings counting from the left side of the chamber) susceptible to stress conditions and wildtype plants (the rest of the seedlings in the chamber) confirms that RoPod provides favorable growth conditions for Arabidopsis plants. Images were acquired using SPIRO. Available in BioRxiv

Video S2. Assessment of root hair length and growth rate in response to sucrose treatment.

Representative video of Col-0 wild-type seedlings grown in RoPods and treated with $0.5 x$ MS (control - left panel) or 0.5x MS supplemented with $1 \%$ sucrose (right panel). Available in BioRxiv

Video S3. Application of the RoPod for time-lapse imaging of pHusion-ATG8a in roots treated with autophagy modulators.

Representative videos of wild-type seedlings expressing the autophagosomal marker pHusionAtATG8a treated with vehicle (0.01\% DMSO), 500 nM Concanamycin A (ConA) or 500 nM AZD 8055 (AZD). ConA blocks the final step of autophagy, i.e. degradation of the autophagic bodies in the vacuole, causing massive accumulation of the pHusion-ATG8-positive puncta in the vacuolar lumen. AZD 8055 induces autophagic activity, causing incorporation of the ATG8a-based reporter into autophagosomes followed by its delivery to the vacuole and degradation. Note decrease of the fluorescent signal after ca $4 \mathrm{~h}$ of treatment. The magenta and green lines indicate respectively hair and non-hair cell files used for quantification. Available in BioRxiv

Video S4. Tutorial to use the semi-automated hair tracking pipeline.

Presentation of the two successive macro used to semi-automatically the root hairs tip in Fiji software. https://github.com/AlyonaMinina/RoPod

Figure S1. Diffusion of chemical compounds in the RoPod. Available in this manuscript

Figure S2. Dynamic changes in the autophagic activity detected in root hair and non-hair cells. Available in this manuscript

Figure S3. Illustration of hair tracking procedure. Available in this manuscript

Supporting methods. Protocol for Arabidopsis growth in RoPods and Ropods printing. Available in this manuscript

File S1. 3D files of RoPods. https://www.alyonaminina.org/ropod-chambers

File S2. Fiji macro used for autophagosome analysis presented in Fig. 3. Available in BioRxiv

File S3. Fiji macro used for root hair tracking analysis presented in Fig. 2. Step1. https://github.com/AlyonaMinina/RoPod

File S4. Fiji macro used for root hair tracking analysis presented in Fig. 2. Step2. https://github.com/AlyonaMinina/RoPod

File S5. Image example used for root hair tracking. The images are for training purpose to use the macros of Supporting information Files S3 and S4. To save space, the size of the images have been reduced compared to the original images actually analyzed. The Supporting information Files S3 and S4 have been adapted to this size reduction. https://github.com/AlyonaMinina/RoPod

\section{References}

[1].Mizushima, N. A brief history of autophagy from cell biology to physiology and disease. Nature Cell Biology 20, 521-527 (2018).

[2].Avin-Wittenberg, T. et al. Review of Autophagy-related Approaches for Improving Nutrient Use Efficiency and Crop Yield Protection. Journal of Experimental Botany (2018) doi:https://doi.org/10.1093/jxb/ery069.

[3].Minina, E. A. et al. Autophagy mediates caloric restrictioninduced lifespan extension in Arabidopsis. Aging Cell 12, (2013).

[4].Minina, E. A. et al. Transcriptional stimulation of ratelimiting components of the autophagic pathway improves plant fitness. Journal of Experimental Botany 69, 1415-1432 (2018). [5].Soto-Burgos, J., Zhuang, X., Jiang, L. \& Bassham, D. C. Dynamics of Autophagosome Formation. Plant Physiology 176, 219-229 (2018).

[6].Börlin, C. S., Lang, V., Hamacher-brady, A. \& Brady, N. R. Agent-based modeling of autophagy reveals emergent regulatory behavior of spatio-temporal autophagy dynamics. 116 (2014). 
bioRxiv preprint doi: https://doi.org/10.1101/2021.12.07.471480; this version posted December $9,2021$. The copyright holder for this preprint (which was not certified by peer review) is the author/funder, who has granted bioRxiv a license to display the preprint in perpetuity. It is made available under aCC-BY-NC-ND 4.0 International license.

[7].Nazio, F. \& Cecconi, F. Autophagy up and down by outsmarting the incredible ULK. Autophagy 13, 967-968 (2017).

[8].Klionsky, D. J. et al. Guidelines for the use and interpretation of assays for monitoring autophagy (3rd edition). Autophagy vol. 12 1554-8635 (Online) (2016).

[9].Dauphinee, A., Ohlsson, J. \& Minina, E. Tandem Tag Assay Optimized for Semi-automated in vivo Autophagic Activity Measurement in Arabidopsis thaliana roots. Bio-Protocol 10, 116 (2020).

[10].Wang, P., Richardson, C., Hawes, C. \& Hussey, P. J. Arabidopsis NAP1 Regulates the Formation of Autophagosomes. Current Biology 26, 2060-2069 (2016).

[11].von Wangenheim, D. et al. Live tracking of moving samples in confocal microscopy for vertically grown roots. eLife 6, (2017).

[12].Grossmann, G. et al. The Rootchip: An integrated microfluidic chip for plant Science. Plant Cell 23, 4234-4240 (2011).

[13].Rongsawat, T., Peltier, J. B., Boyer, J. C., Véry, A. A. \& Sentenac, H. Looking for Root Hairs to Overcome Poor Soils. Trends in Plant Science 26, 83-94 (2021).

[14].Jain, A. et al. Differential effects of sucrose and auxin on localized phosphate deficiency-induced modulation of different traits of root system architecture in Arabidopsis. Plant Physiology 144, 232-247 (2007).

[15].Datta, S., Prescott, H. \& Dolan, L. Intensity of a pulse of RSL4 transcription factor synthesis determines Arabidopsis root hair cell size. Nature Plants 1, 1-6 (2015).

[16].Baluška, F. et al. Root hair formation: F-actin-dependent tip growth is initiated by local assembly of profilin-supported Factin meshworks accumulated within expansin-enriched bulges.
Developmental Biology 227, 618-632 (2000).

[17].Grierson, C., Nielsen, E., Ketelaarc, T. \& Schiefelbein, J. Root Hairs. The Arabidopsis Book 12, e0172 (2014).

[18].Carol, R. J. et al. A RhoGDP dissociation inhibitor spatially regulates growth in root hair cells. Nature 438, 1013-1016 (2005).

[19].Denninger, P. et al. Distinct RopGEFs Successively Drive Polarization and Outgrowth of Root Hairs. Current Biology 29, 1854-1865.e5 (2019).

[20].Marshall, R. S. \& Vierstra, R. D. Autophagy: The Master of Bulk and Selective Recycling. Annual Review of Plant Biology 69, 173-208 (2018).

[21].Dauphinee, A. N. et al. Chemical screening pipeline for identification of specific plant autophagy modulators. Plant Physiology 181, 855-866 (2019).

[22].Hachez, C. et al. Arabidopsis SNAREs SYP61 and SYP121 coordinate the trafficking of plasma membrane aquaporin PIP $2 ; 7$ to modulate the cell membrane water permeability. Plant Cell 26, 3132-3147 (2014).

[23].Kirchhelle, C. \& Moore, I. A simple chamber for long-term confocal imaging of root and hypocotyl development. Journal of Visualized Experiments 2017, 1-9 (2017).

[24].Clark, N. M. et al. Novel Imaging Modalities Shedding Light on Plant Biology: Start Small and Grow Big. Annual Review of Plant Biology 71, 789-816 (2020).

[25].Curtis, M. D. \& Grossniklaus, U. A Gateway Cloning Vector Set for High-Throughput Functional Analysis of Genes in Planta. Plant physiology 133, 462-469 (2003).

[26].Schindelin, J. et al. Fiji: An open-source platform for biological-image analysis. Nature Methods 9, 676-682 (2012).

[27].Berg, S. et al. Ilastik: Interactive Machine Learning for (Bio)Image Analysis. Nature Methods 16, 1226-1232 (2019). 


\section{Supporting Figures}

a

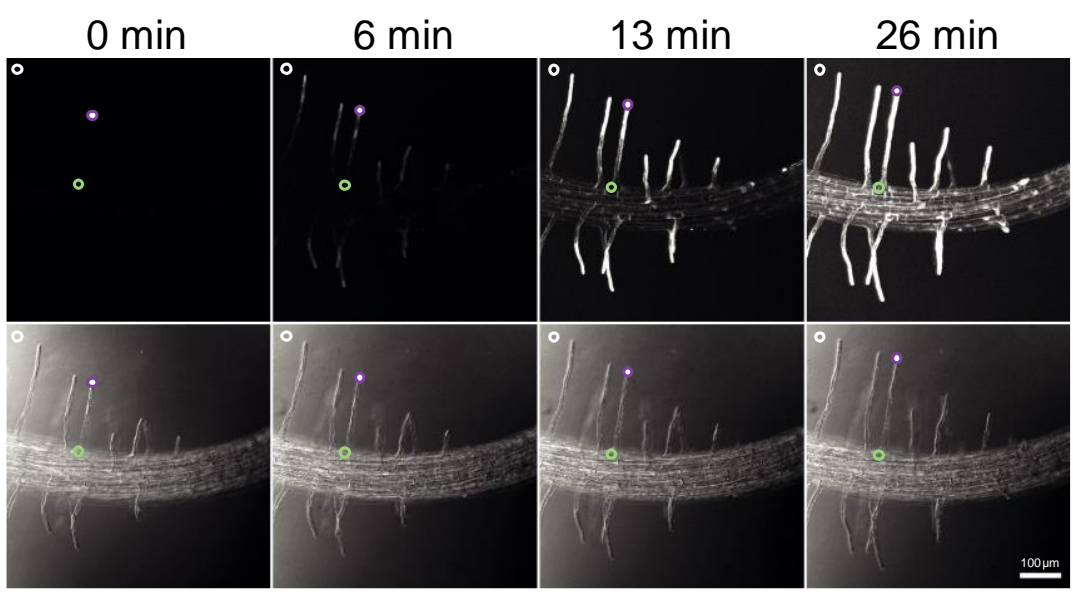

b

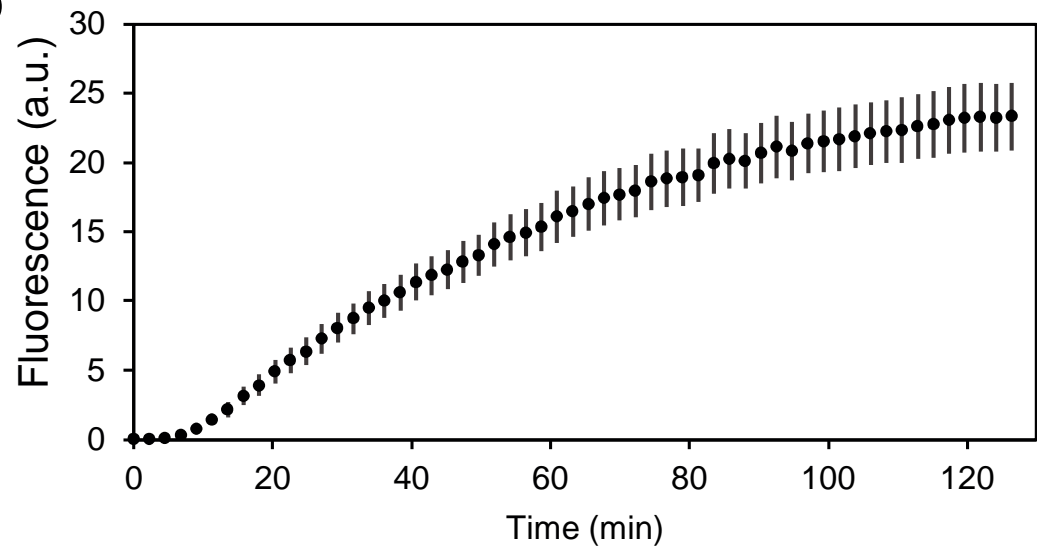

\section{Supporting information Figure S1. Diffusion of chemical compounds in the RoPod.}

(a) Representative time lapse images of roots treated with fluorescein in the RoPod. Arabidopsis seedlings were grown in RoPods using the described protocol and liquid MS medium containing $2 \mu \mathrm{g}$ $\mathrm{ml}^{-1}$ of fluorescein dye was added to the chambers immediately prior to start of imaging. Maximum intensity projections of the fluorescent signal is shown on the top row, corresponding transmitted light images are shown in the bottom row. The circles indicate representative regions of interest analysed for each root hair to produce the data presented in Fig. 1f. Magenta circle, root hair tip; green circle, root hair base; white circle, background.(b) Dynamics of fluorescence accumulation at the bottom coverslip of RoPod. In this experiment liquid MS medium containing $140 \mu \mathrm{g} \mathrm{ml}^{-1}$ fluorescein was added to RoPods containing only growth medium. The fluorescence was recorded in four separate chambers, with 2 to 3 fields of view chosen at random positions in each RoPod. 


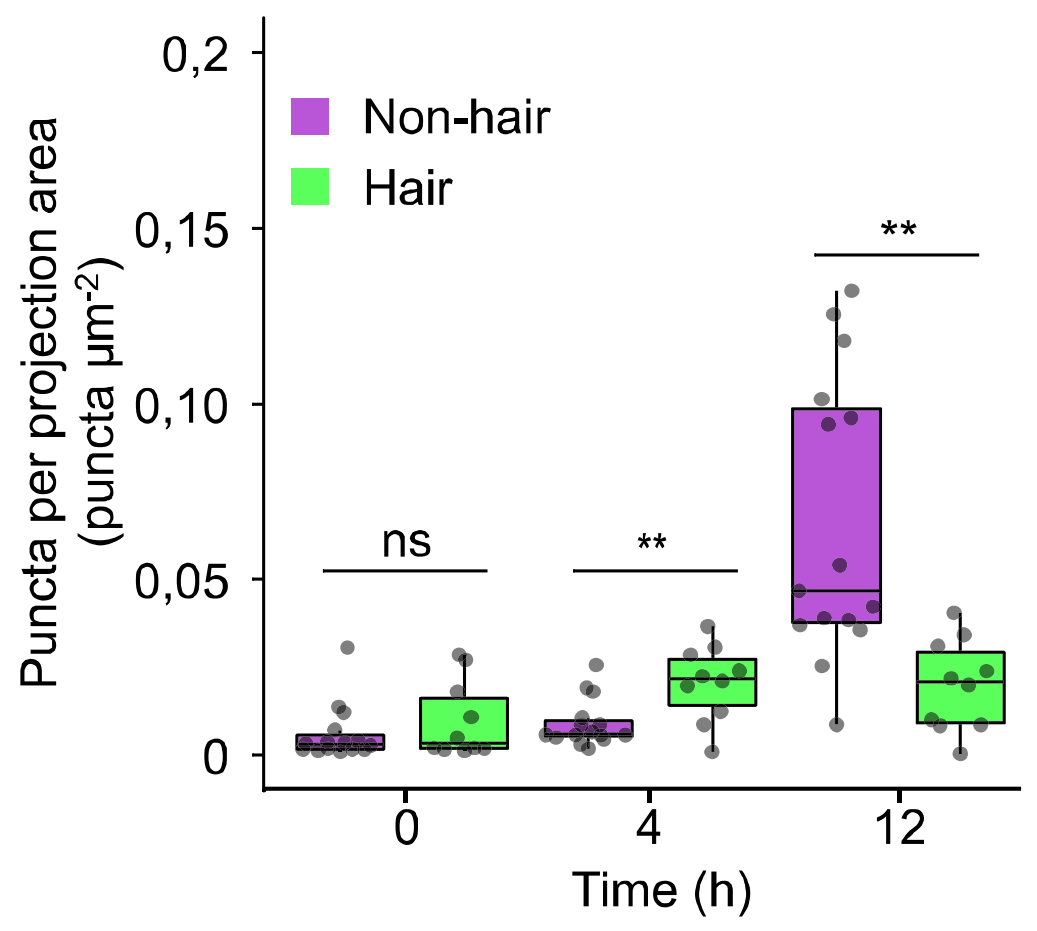

Supporting information Figure S2. Dynamic changes in the autophagic activity detected in root hair and non-hair cells.

Quantification of pHusion-ATG8-positive puncta per area in the epidermal root cells of wild-type seedlings grown in RoPods and treated with $500 \mathrm{nM}$ ConA. The data represents $0 \mathrm{~h}, 4 \mathrm{~h}$ and $12 \mathrm{~h}$ time points of the time-lapse assays shown in the Fig. 2 and video S3. T-test, $\mathrm{P}<0.01$. 
bioRxiv preprint doi: https://doi.org/10.1101/2021.12.07.471480; this version posted December 9, 2021. The copyright holder for this preprint (which was not certified by peer review) is the author/funder, who has granted bioRxiv a license to display the preprint in perpetuity. It is made available under aCC-BY-NC-ND 4.0 International license.

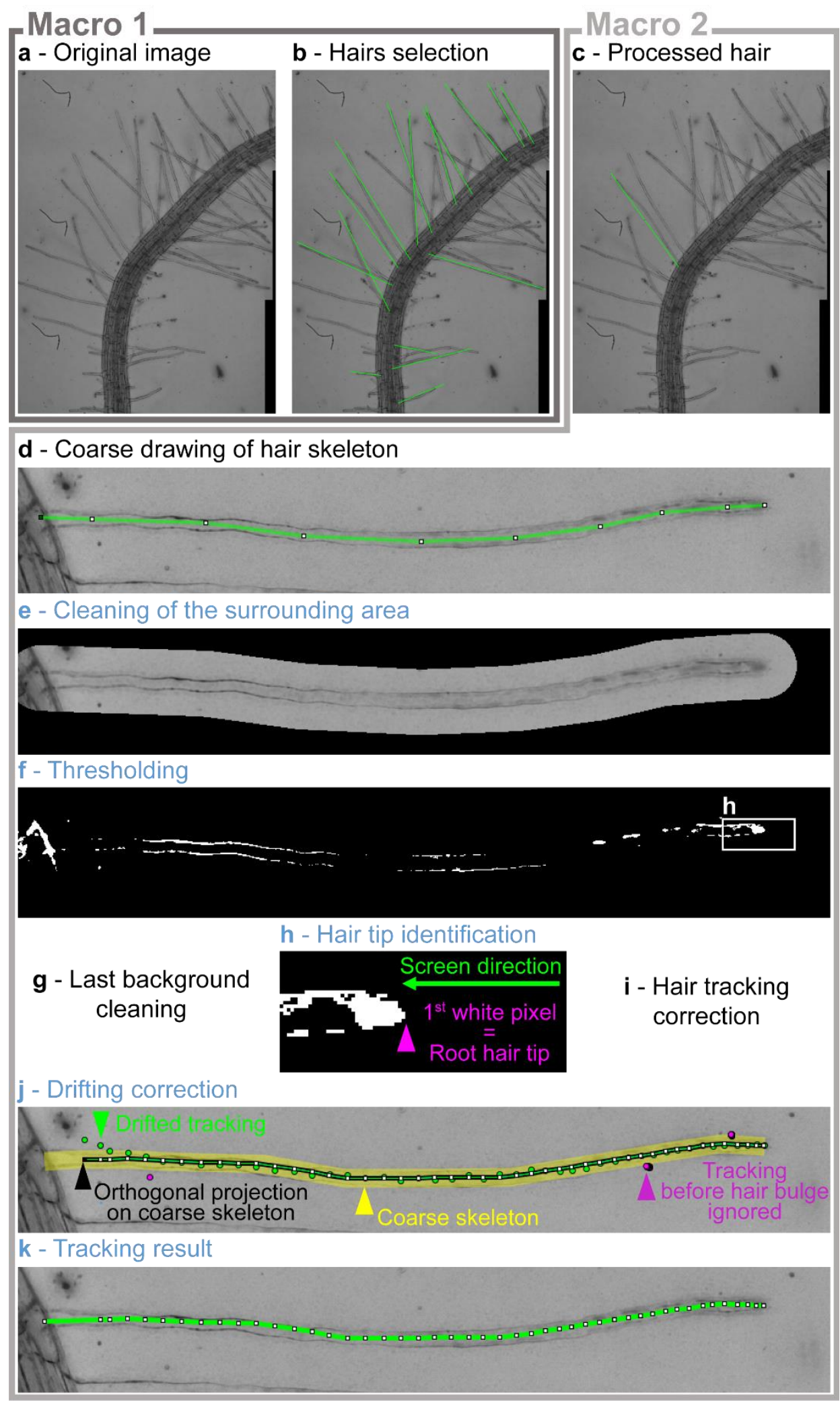

Supporting information Figure S3. Illustration of the hair tracking procedure.

(a-b) Macro 1: Selection of root hairs for analysis. (c-k) Macro 2: Tracking of selected root hairs. The steps indicated in blue are automatic steps. The selection in $\mathbf{f}$ is increased in $\mathbf{h}$. 
bioRxiv preprint doi: https:/doi.org/10.1101/2021.12.07.471480; this version posted December 9, 2021. The copyright holder for this preprint (which was not certified by peer review) is the author/funder, who has granted bioRxiv a license to display the preprint in perpetuity. It is made available under aCC-BY-NC-ND 4.0 International license.

\section{Supporting methods}

\section{Protocol for Arabidopsis growth in RoPods}

Shown here on the example of RoPod 5

Please note that updates are published on the website:

https://www.alyonaminina.org/ropod

In this video you can find a short step-by-step demonstration of how to prep RoPod chambers and grow plants in them.

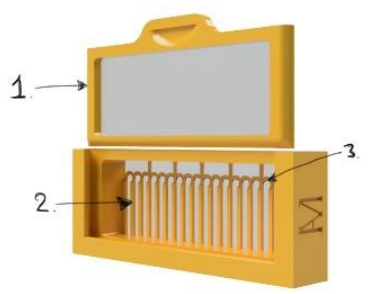

\section{RoPod 5 has:}

1. A watertight lid that locks by curving out. To unlock the lid, gently push its handle upwards and slide the lid inside its slot

2. Arcs to guide roots into the channels

3. Separators to prevent neighbouring roots from crossing and create "lanes" for individual root growth.

\section{Step 1.}

Sterilize the RoPod under UV in a laminar flow hood. Place the open chamber and the lid (inner side up) under the UV light, $30 \mathrm{~min}$ of exposure is usually sufficient.

Chambers can also be sterilized by submerging in $70 \%$ Ethanol for $30 \mathrm{~min}$ and then rinsing in 95\% Ethanol and leaving to air dry in a laminar flow hood. However, in this case it is crucial to ensure that ethanol completely evaporated as even traces of it will have adverse effect on seedling growth.

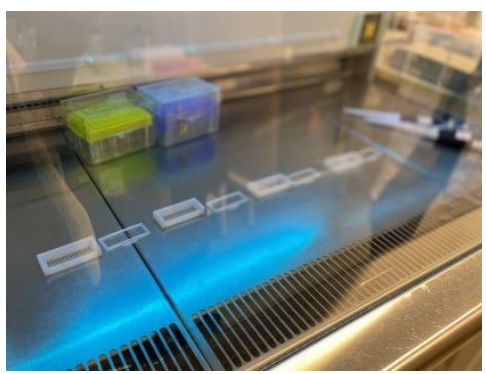

\section{Step 2.}

Thaw $0.5 \times \mathrm{MS}$ in a microwave, let it cool to $60 \mathrm{C}$ and pipette $3 \mathrm{~mL}$ into the sterile chamber. Make sure the medium is evenly distributed in the chamber and there are no bubbles

\section{Recommended $\mathbf{V}$ of the medium:}

$1 \mathrm{~mL}$ of medium per a well of the 3 -well chamber, i.e.

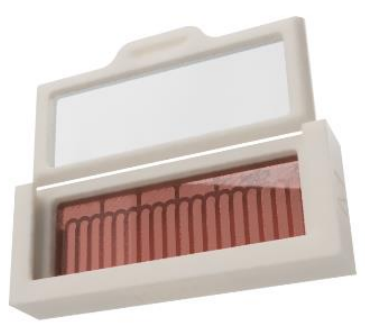
RoPod 3

$1.5 \mathrm{~mL}$ well of a 2-well chamber, i.e. RoPod 2

$3 \mathrm{~mL} / 1$-well chamber, i.e. RoPod 1 and 5

\section{$0.5 \times$ MS:}

$0.5 x$ MS including vitamins (M0222, Duchefa)

10 mM MES (M1503, Duchefa)

$1 \%$ sucrose

$0.8 \%$ Plant agar (P1001, Duchefa)

Adjust $\mathrm{pH}$ with $\mathrm{KOH}$ to 5.8

Autoclaved for $20 \mathrm{~min}$ at $120^{\circ} \mathrm{C}$

Store at $4 \mathrm{C}$ 


\section{Step 3.}

Let the medium solidify and then use a tip or toothpick to remove a ca $5 \mathrm{~mm}$ wide strip of the medium, this will give space for the areal part of seedlings. For RoPod 5 make sure arcs are exposed.

\section{Step 4.}

Transfer sterile seeds using a sterile toothpick onto the bottom glass by the edge of the medium. For RoPod 5 place the seeds "under" the arches, which are designed to guide roots of young seedling into the individual channels.

Seeds can be surface sterilized in $70 \% \mathrm{EtOH} / 0.05 \%$ TritonX-100 for 20 min, washed three times with $96 \%$ $\mathrm{EtOH}$ and air dried in a laminar flow hood. Sterile seeds can be stored for up to half a year.

Please note, that RoPod chambers with printed in glass have a $0.5 \mathrm{~mm}$ plastic rim surrounding the coverslip glass, which might not allow to image with immersion objectives very close to the edges of the glass window. If you plan to use $40 x$ and higher magnifications, it is best to not image in the first and last lanes of RoPod 5 .

\section{Step 5.}

Close the chamber. RoPods with watertight lids do not need to be sealed individually, other RoPods require it. Place the chamber into a square Petri plate and seal it.

\section{Step 6.}

Place the plate under growth conditions, vertically or slightly tilted to guide root growth towards the coverslip bottom
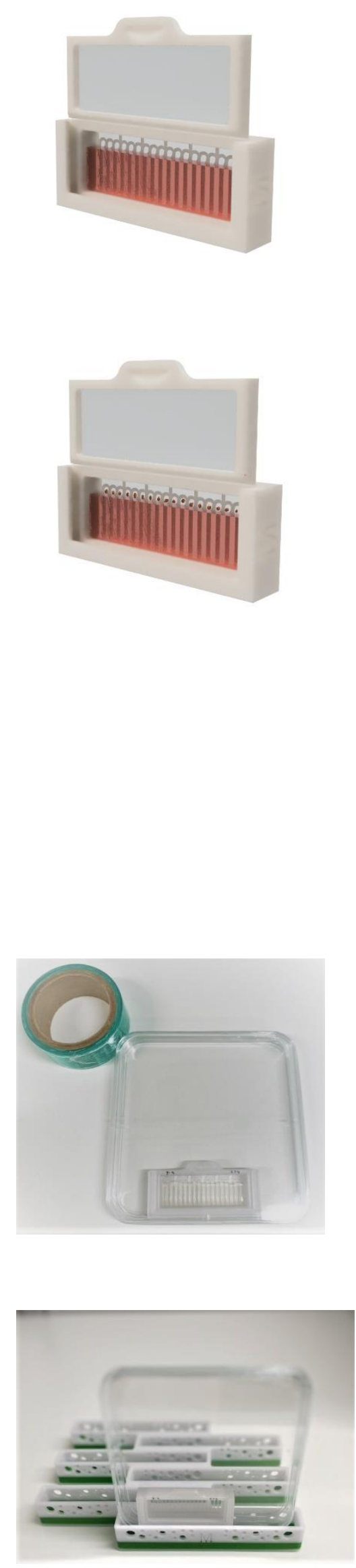
bioRxiv preprint doi: https://doi.org/10.1101/2021.12.07.471480; this version posted December 9, 2021. The copyright holder for this preprint (which was not certified by peer review) is the author/funder, who has granted bioRxiv a license to display the preprint in perpetuity. It is made available under aCC-BY-NC-ND 4.0 International license.

\section{Protocol for RoPods printing}

Please note that updates are published on the website:

https://www.alyonaminina.org/ropod

In this video you will find a quick step-by-step demonstration how to print a RoPod

For RoPods 1-5 microscopy cover glass is printed into the plastic, thus resolving the issues with its detachment during experiments and reuse.

\section{RoPod 5}

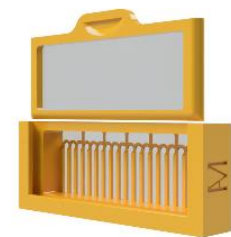

- Water tight lid

- Channels for individual roots

- Can accommodate up to 18 bio replicates

- Arcs to guide agravitropically growing roots into their channels

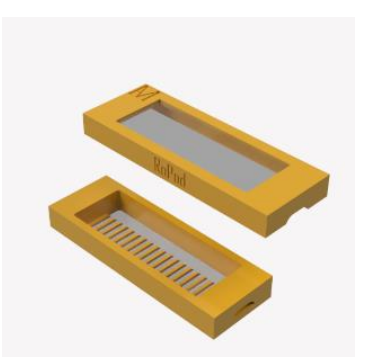

\section{RoPod 4}

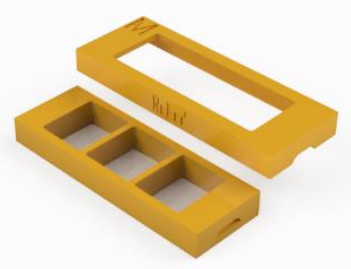

\section{RoPod 3}

- The lid is easy to remove and place back, for adding treatments during imaging

- 3 wells for growing different genotypes

- Separators between wells are not watertight

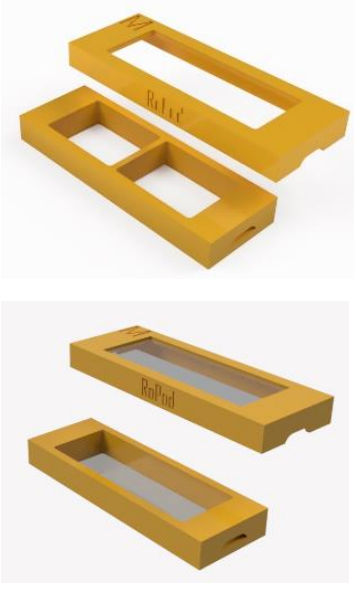

\section{RoPod 2}

- The lid is easy to remove and place back, for adding treatments during imaging

- 2 wells for growing different genotypes

- Separators between wells are not watertight

\section{RoPod 1}

- is easy to remove and place back, for adding treatments during imaging

- 1 well with no separators, can be used to grow roots along the longer axis

- Can be also used for mounting samples in a humid chamber instead of using a sample glass and cover slip

\section{Commercially available chambers tested for RoPod protocol:}

- Ibidi chambered coverslips (cat \# 80421 and 80286 \# 1.5 polymer coverslip, hydrophobic, sterilized, Ibidi, Germany)

- 1-well II Chamber Slide ${ }^{\mathrm{TM}}$ System (Nunc ${ }^{\mathrm{TM}}$ Lab-Tek $^{\mathrm{TM}}$, cat \# C6307, SigmaAldrich)

- x-well cell culture chamber, 1-well, on coverglass (cat \# 94.6190.102, Sarstedt). 


\section{For printing RoPods you will need:}

- access to a 3D printer extruder with a nozzle $0.4 \mathrm{~mm}$ (If you do not have access to a printer, consider finding Makerspace facility in your vicinity)

- PETG filament, white or transparent, $\varnothing 1.75 \mathrm{~mm}$

- 24 x $60 \mathrm{~mm}$ microscopy coverslip \#1.5, i.e. 0.16-0.19 mm thick ( $\mathrm{VWR}, 630$ 2108).

- Superglue

- $.3 m f$ or .stl file for RoPods

\section{Step 1.}

- It is highly recommended to generate from the provided $.3 \mathrm{mf}$ project files. However, we also provide.stl files

- If you prefer to use .stl, set the following parameters:

- follow the instructions for the chamber positioning

- Print settings-> Layer height $=0.1$ $\mathrm{mm}$

- Print settings-> Fill density $=50 \%$

- Print settings-> Fill pattern $=3 \mathrm{D}$ Honeycomb

- Print settings-> Layers and Perimeters-> Vertical shells-> Perimeters $=4$

- Slice the model and insert a pause after $5 \mathrm{~mm}$ height

- You can simultaneously print up to 17 RoPods on a Prusa MK2or MK3. However we strongly recommend to start with just one

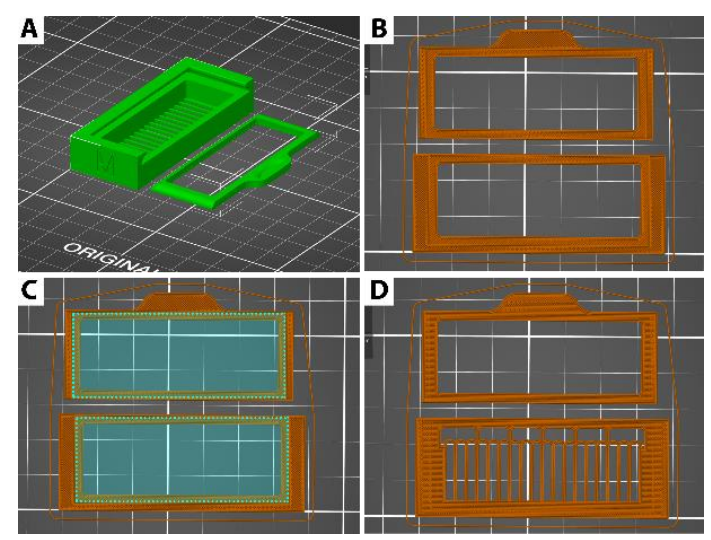

A. The RoPod 5 chamber and the lid should both be upside up on the print bed.

B. The layer with the slot for glasses should be the last one before the pause

C. Slot for the glasses is highlighted with the dotted line

D. The very next layer after the paused layer

\section{Step 2. Clean the coverslip glasses}

- Remove dust and fat from the glasses by wiping them clean with a paper tissue soaked in acetone

\section{Step 3. Start the print}

- Start the print and wait until it automatically pauses on the layer programmed int the Step 1.

- The extruder will move to the back left of the print bed while waiting for the user to

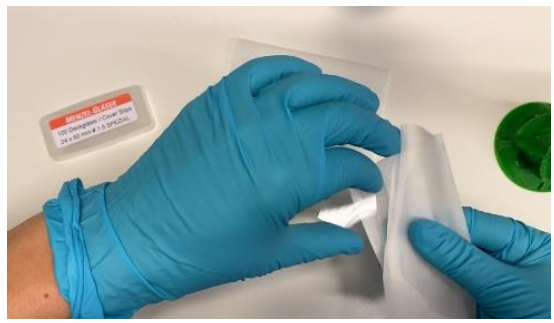
resume the print 


\section{Step 4. Place the glasses into the slots}

- The chambers and lids models have $0.1 \mathrm{~mm}$ deep slots for the coverslips

- Add a drop of super glue to two corners of a glass. This will secure the glass' position during printing

- Use forceps and patience to carefully place the glass into its slot. Make sure it does not stick upwards or sideways

- Press down on the the corners with glue and hold for a few seconds. sic! wear gloves! superglueing oneself to a 90C- hot print bed is not a good way to start your day

\section{Step 5. Resume the print}

- Resume the print and make sure the the filament does not move the glass from its position and does not crack it

- When the chamber is ready make sure there is no filament sticking sideways. If needed imperfections can be removed with a scalpel
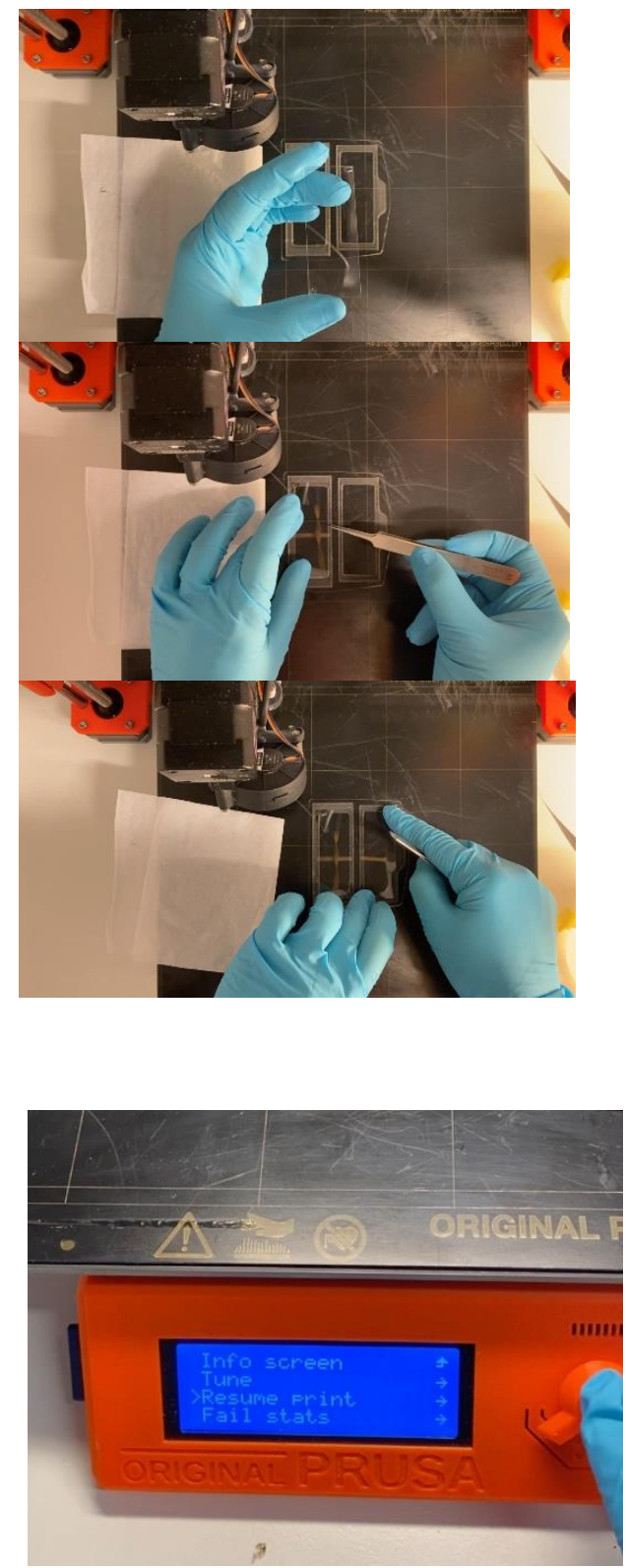
bioRxiv preprint doi: https://doi.org/10.1101/2021.12.07.471480; this version posted December 9, 2021. The copyright holder for this preprint (which was not certified by peer review) is the author/funder, who has granted bioRxiv a license to display the preprint in perpetuity. It is made available under aCC-BY-NC-ND 4.0 International license.

\section{Supporting files}

File S1. 3D files and printing instructions of RoPods.

File name: Supporting information File S1_3D files.zip

Location: https://www.alyonaminina.org/ropod-chambers

File S2. Fiji macro used for autophagosome analysis presented in Fig. 3.

File name: Supporting information File S2_Autophagosome analysis.ijm Location: Available in BioRxiv

File S3. Fiji macro used for root hair tracking analysis presented in Fig. 2. Step1.

File name: Supporting information File S3_Root hair tracking_step1.ijm

Location: https://github.com/AlyonaMinina/RoPod

File S4. Fiji macro used for root hair tracking analysis presented in Fig. 2. Step2.

File name: Supporting information File S4_Root hair tracking_step2.ijm

Location: https://github.com/AlyonaMinina/RoPod

File S5. Image example used for root hair tracking. The images are for training purpose to use the macros of Supporting information Files $\mathbf{S 3}$ and S4. To save space, the size of the images have been reduced compared to the original images actually analyzed. The Supporting information Files S3 and S4 have been adapted to this size reduction.

File name: Supporting information File S5_Image example for root hair tracking training.zip Location: https://github.com/AlyonaMinina/RoPod 